\title{
MEASURING FEL RADIATION PROPERTIES AT VISA-FEL
}

\author{
A. Murokh*, R. Agustsson, P. Frigola, C. Pellegrini, S. Reiche, J. Rosenzweig, A. Tremaine, \\ University of California, Los Angeles, CA 90095 \\ M. Babzien, I. Ben-Zvi, E. Johnson, R. Malone, G. Rakowsky, J. Skaritka, X.J. Wang, \\ BNL, Upton NY 11973 \\ K.A. Van Bibber, L. Bertolini, J.M. Hill, G.P. Le Sage, M. Libkind, A. Toor, \\ LLNL, Livermore, CA 94551 \\ R. Carr, M. Cornacchia, L. Klaisner, H.-D. Nuhn, R. Ruland, SLAC, Stanford, CA 94309
}

\begin{abstract}
The VISA (Visible to Infrared SASE Amplifier) SASE free electron laser has been successfully operated at the Accelerator Test Facility (ATF) at BNL. High gain and saturation were observed at $840 \mathrm{~nm}$. We describe here the diagnostic system, experimental procedures and data reduction algorithms, as the FEL performance was measured along the length of the undulator. We also discuss selected spectral radiation measurements.
\end{abstract}

\section{INTRODUCTION}

The VISA experiment started in 1998, with the objective of studying SASE-FEL physics at visible to near IR frequency range [1]. The linac RF system at the ATF was upgraded for VISA, to produce a high brightness 71 $\mathrm{MeV}$ electron beam. To achieve the maximum performance, a 4-m Hallbach array undulator was designed with a strong focusing periodic lattice [2]. Given the sub-100 $\mu \mathrm{m}$ tolerance on the electron beam trajectory walk-off [3], such a design places stringent limitations on the error budget of the undulator correction and alignment $[4,5]$.

The experimental base was developed through the year 2000 , and in the Spring 2001 saturation at $3.6 \mathrm{~m}$ was measured with a power gain length of $18.7 \mathrm{~cm}$. A more complete overview of the experiment is given in [6], whereas this paper is focused on the measurement procedures and the data analysis techniques.

\section{INTRA-UNDULATOR DIAGNOSTICS}

The undulator and vacuum chamber design [7] include openings for 8 diagnostics ports on both sides of the chamber, spaced $50 \mathrm{~cm}$ apart, so that the electron beam information as well as the FEL radiation can be extracted all the way throughout the length of the undulator. The major challenge was to build the diagnostic probes that fit into the narrow 3.7-mm aperture in the undulator channel. The undulator probes (Fig. 1) were developed to insert the active area into the undulator gap and transport the light out through the periscope principle. A light aluminum frame locks the triangular 2 -sided $45^{\circ}$ mirror. The probe has two positions controlled by the actuator: (1) when the outer side of the mirror intercepts the electron beam and

*murokh@physics.ucla.edu

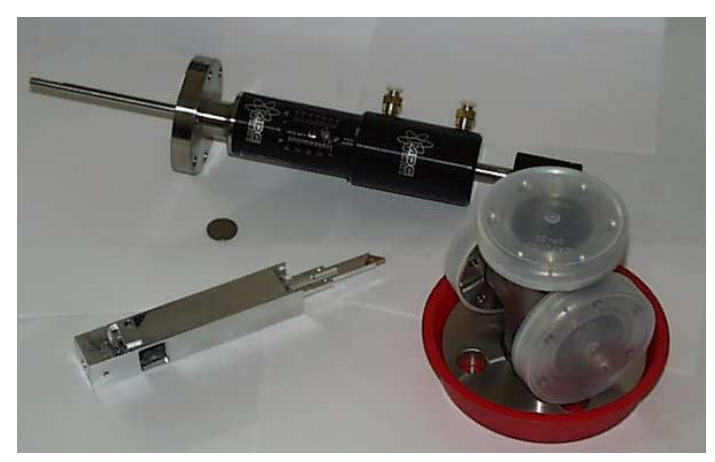

Figure 1: Main components of the intra-undulator diagnostic probe assembly.

extracts the FEL radiation; and (2) when the inner side of the mirror deflects OTR from the electron beam through the periscope into the imaging system. An ASA flange allows the careful positioning of the probe to avoid contact with the undulator magnets. A number of modifications to the original design took place as the experiment evolved [8]. In the final configuration silicon mirrors are used at the probe tips. Silicon is a brittle material and the mirrors developed many cracks, yet it was the only material that can be polished to a laser finish, given the tiny size of the mirrors $(2.2 \times 10 \mathrm{~mm})$.

A detailed discussion on the challenges of the electron beam imaging was presented in [8]. The system operation was successful for the rough trajectory alignment. Although the electron beam profile was measured under

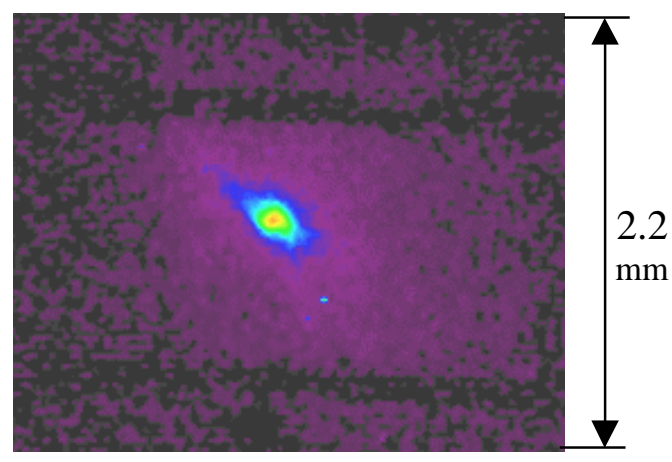

Figure 2: OTR image of the electron beam inside the undulator. The measured beam size is $120 \mu \mathrm{m}$ FWHM. 
certain conditions (Fig. 2); a small dynamic range of the OTR and a high optical noise background did not allow careful measurements of the electron beam envelope dynamics throughout the undulator. In addition, beam fluctuations due to the RF jitter made systematic electron beam measurements rather difficult inside the undulator.

FEL diagnostics was positioned on the opposite side of the probe actuators assembly. The light deflected by the outer side of the probe silicon mirror follows through the relay imaging transport line towards the diagnostics, whether there is a joulemeter, CCD camera, or a spectrometer. Flipper based mirrors can be inserted on the optical beam path to allow multiplexing between the ports [8].

To measure charge simultaneously with the joulemeter readout, a stripline beam position and charge monitor (BPM) was installed in front of the VISA undulator. A BPM sum signal is mixed with a $1 \mathrm{GHz}$ reference signal to produce a readable slow signal with a peak-to-peak value linearly proportional to the electron beam charge. The signal was periodically calibrated against the Faraday cup located downstream of the undulator. Typical linear dependence indicated that there was no significant charge loss near the operating point of the FEL. The signals from the BPM and a joulemeter were displayed on the oscilloscopes, and the GPIB protocol was developed to enable shot-by-shot simultaneous recording of both signal values.

\section{RESULTS AND ANALYSIS}

\subsection{Exponential gain}

The operating procedure for the exponential gain measurements starts with the electron beam trajectory optimization at the photo-injector beam line. It is most essential to steer the beam through the center of the linac sections so that it can be properly matched into the transport focusing channel (Fig. 3) pre-tuned from the previous experimental run. Through the dispersive section the electron beam is being transported towards the experimental area, where it is matched into the undulator entrance. The beamline tune is set to compensate for the linear dispersion in the system. Once the beam is steered through the center of the undulator, one can find an optimal RF phase of the linac, where some gain can be observed. Then the system is fine-tuned based on the gain until the satisfactory operating point is found. To keep the

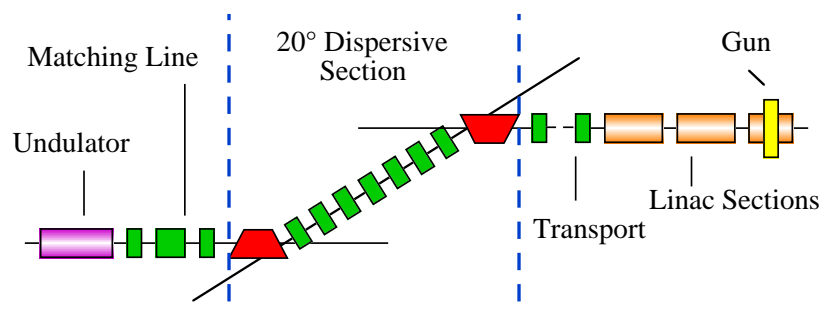

Figure 3: VISA beam line layout at ATF.
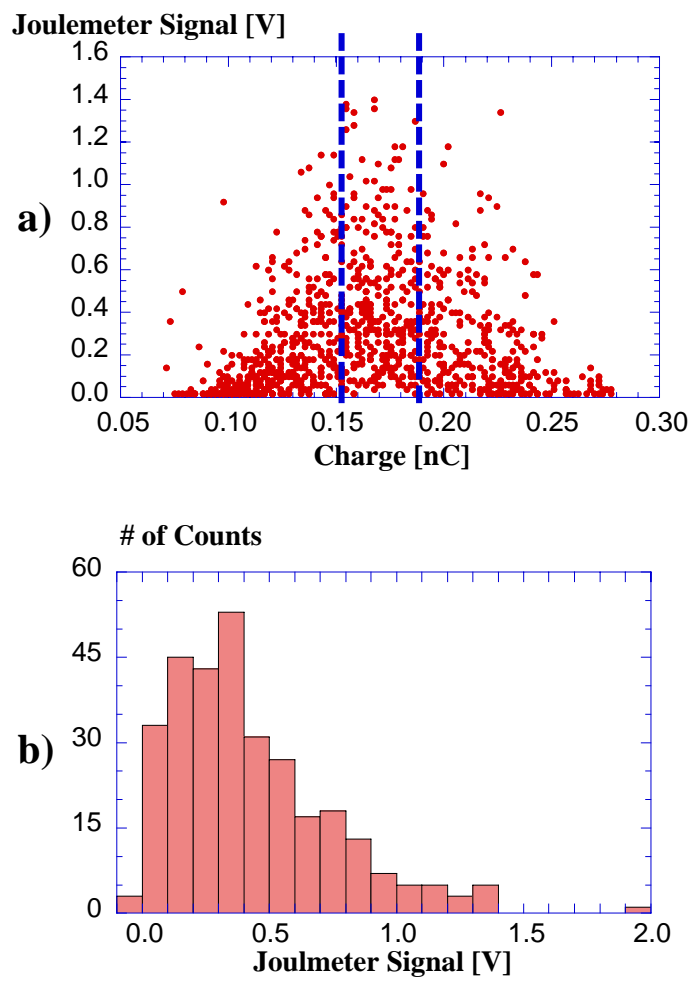

Figure 4: (a) FEL pulse energy measured with respect to the charge for 1500 consecutive shots at pop-in 8; (b) statistical distribution of the pulse energies within the $20 \%$ charge cut window.

FEL at the operating point for the duration of the measurement one has to occasionally readjust the $\mathrm{RF}$ phase of the gun, which slowly drifts throughout the day. It is not possible to prevent a shot-to-shot jitter of the linac RF phase which has a dramatic effect on the laser performance, as it will be shown below.

In Fig. 4a one can see a typical shot-to-shot SASE intensity distribution measured $25 \mathrm{~cm}$ before the end of the undulator, at the system operating point. So called "Christmas tree" shape of this distribution has rather anomalous nature, and we believe is originated in the linac phase drift. At the right hand side of the plot, the electron beam rides on the top of the linac phase and all the charge propagates through the undulator. Linac phase drift introduces a chirp to the beam, which leads to a charge loss in the $1.5 \mathrm{~cm}$ bending magnet aperture located in the middle of the dispersive section. This causes dramatic charge fluctuations over a $1^{\circ}$ span of the linac phase. However, as the chirp increases, the beam undergoes a strong longitudinal compression at the dispersive section ${ }^{1}$, which improves the FEL lasing despite the overall charge loss. On the left hand side of the "Christmas tree" charge loss and overcompression lead to the FEL performance degradation.

\footnotetext{
1 This statement is supported by the most recent CTR bunch length measurements performed at the undulator exit. Detailed analysis of these results will be presented in the future publications.
} 


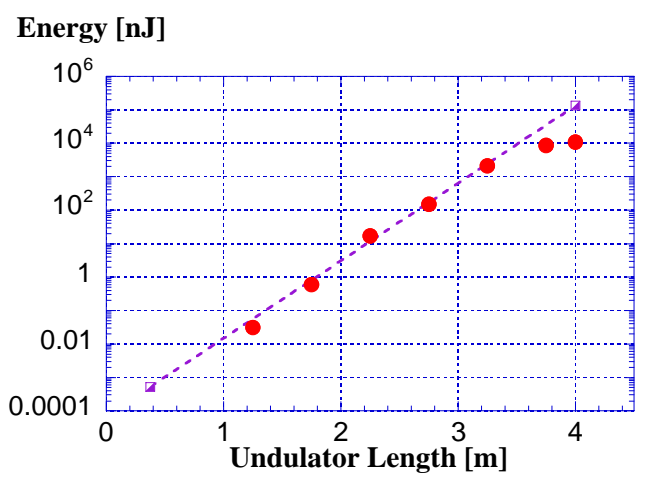

Figure 5: Example of the FEL intensity versus length measurement. Dashed curve represents the fit to the exponential part of the curve.

Even though it is not possible to simultaneously measure the FEL intensity at the different points along the undulator, we can compare the distributions measured at the different undulator ports at different times, but at the same operating point. Indeed, the anomalous scraping effect described above, serves to our benefit - as the measured beam charge marks a jittery linac phase with high accuracy. While comparing the data sets collected at the different locations along the undulator, we consider only the data points which appear in a narrow charge window at the peak of the distribution (see Fig. 4b), and therefore fall into the same linac operating point. This selection rule enabled a valid comparison of the data sets collected along the undulator length. On Figure 5, each point on the curve represents simply the top $10 \%$ of the distributions, an example of which is shown on Figure $4 \mathrm{~b}$. The exponential fit to the first 5 points on the curve yields a power gain length of $18.7 \mathrm{~cm}$. More systematic statistical analysis generates similar value for the gain length, yet it is beyond the scope of this paper. The last 2 points on the curve indicate saturation. The overall saturation gain can be calculated by extrapolating an exponential curve down to the one field gain length point past the undulator entrance. This point can be considered to be a seed of the FEL process [9], yielding a gain value of $2.2 \times 10^{7}$. It is noteworthy here, that given the possible effect of the bunch length compression mentioned here before, the system may fall into the short bunch regime [10] where a more complex treatment may be required.

\subsection{Spectral Measurements}

The spectral measurements have been performed with a miniature fiber spectrometer, calibrated by a manufacturer and independently tested with the diode lasers. The spectrometer resolution is $1.5 \mathrm{~nm}$, with a dynamic range $350-1000 \mathrm{~nm}$. Calibrated neutral density filters where used to compare spectra of the SASE light through the large range of signal intensities.

Before the high gain system operating point was found, we performed spectral measurements at rather low gain

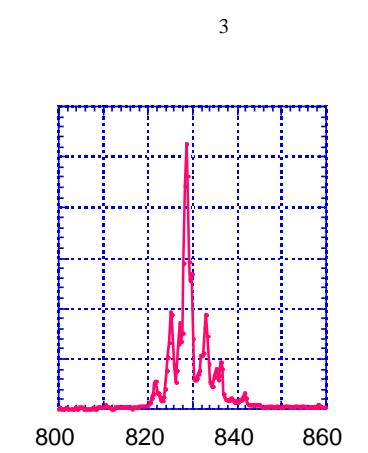

6

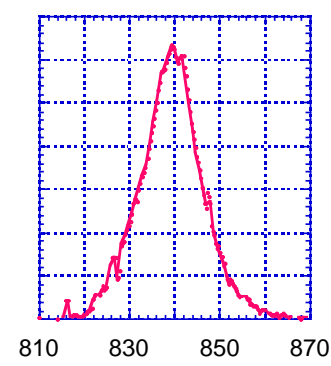

Figure 6: Comparing spectrum of the fundamental measured at the end of the undulator for the cases of low and high gain.

$(\mathrm{G} \sim 500)$. The typical spectrum profile contained many spikes (Fig. 6 left) indicating a long bunch limit, in good agreement with the $300-400 \mu \mathrm{m}$ RMS bunch length measured in the linac.

In the present high gain operation regime the measured spectrum lacks structure (Fig. 6 right), indicating very few spikes in the temporal profile [10], which would be a consequence of the strong bunch compression in the dispersive section.

\section{CONCLUSION}

We developed a diagnostic system to examine a SASE beam properties of the VISA FEL. Intra-undulator FEL diagnostics allowed measurements of the exponential gain and saturation. The data reduction routine was developed to systematically analyze data sets obtained over the time periods much longer than the typical time of the system stable operation. In-depth analysis of all the data accumulated throughout the recent experimental period will be reported in the near future. This work was supported by DOE contract No DE-FG03-92ER40693 and the LCLS collaboration.

\section{REFERENCES}

[1] http://www-ssrl.slac.stanford.edu/VISA

[2] R. Carr et al, Submitted to Phys. Rev. Letters

[3] P. Emma, H.D. Nuhn, FEL 98 Proceedings II, 35-36 (1999)

[4] R. Ruland et al, PAC 99 Proceedings, 1390-1392 (1999)

[5] G. Rakowsky et al, PAC 99 Proceedings, 2698-2700 (1999)

[6] A. Tremaine et al, the same Proceedings

[7] M. Libkind et al, PAC 99 Proceedings, 2477-2479 (1999)

[8] A. Murokh et al, FEL 2000 Proceedings (2001)

[9] L. Yu and S. Krinsky, Nucl. Instr. and Meth. A 285 119-121 (1989)

[10] R. Bonifacio et al, Phys. Rev. Letters 73(1), 70-73 (1994) 